OPEN ACCESS

Edited by:

Yun-Qing Li,

The Fourth Military Medical

University, China

Reviewed by:

Malgorzata Maria Kossut,

Nencki Institute of Experimental

Biology, Poland

Michael Lardelli,

University of Adelaide, Australia

${ }^{*}$ Correspondence: Ursula H. Winzer-Serhan uwserhan@tamhsc.edu

Received: 13 October 2015

Accepted: 30 October 2015

Published: 18 November 2015

Citation:

Damborsky JC, Slaton GS and Winzer-Serhan UH (2015) Expression of Npas4 mRNA in Telencephalic Areas of Adult and Postnatal Mouse Brain

Front. Neuroanat. 9:145. doi: 10.3389/fnana.2015.00145

\section{Expression of Npas4 mRNA in Telencephalic Areas of Adult and Postnatal Mouse Brain}

\author{
Joanne C. Damborsky, G. Simona Slaton and Ursula H. Winzer-Serhan* \\ Department of Neuroscience and Experimental Therapeutics, Texas A\&M University System Health Science Center, \\ Bryan, TX, USA
}

The transcription factor neuronal PAS domain-containing protein 4 (Npas4) is an inducible immediate early gene which regulates the formation of inhibitory synapses, and could have a significant regulatory role during cortical circuit formation. However, little is known about basal Npas4 mRNA expression during postnatal development. Here, postnatal and adult mouse brain sections were processed for isotopic in situ hybridization using an Npas4 specific cRNA antisense probe. In adults, Npas4 mRNA was found in the telencephalon with very restricted or no expression in diencephalon or mesencephalon. In most telencephalic areas, including the anterior olfactory nucleus $(\mathrm{AON})$, piriform cortex, neocortex, hippocampus, dorsal caudate putamen (CPu), septum and basolateral amygdala nucleus (BLA), basal Npas4 expression was detected in scattered cells which exhibited strong hybridization signal. In embryonic and neonatal brain sections, Npas4 mRNA expression signals were very low. Starting at postnatal day 5 (P5), transcripts for Npas4 were detected in the AON, CPu and piriform cortex. At P8, additional Npas4 hybridization was found in CA1 and CA3 pyramidal layer, and in primary motor cortex. By P13, robust mRNA expression was located in layers $\mathrm{IV}$ and $\mathrm{VI}$ of all sensory cortices, frontal cortex and cingulate cortex. After onset of expression, postnatal spatial mRNA distribution was similar to that in adults, with the exception of the $\mathrm{CPu}$, where Npas4 transcripts became gradually restricted to the most dorsal part. In conclusion, the spatial distribution of Npas4 mRNA is mostly restricted to telencephalic areas, and the temporal expression increases with developmental age during postnatal development, which seem to correlate with the onset of activity-driven excitatory transmission.

Keywords: telencephalon, LE-PAS, cortex, hippocampus, development, in situ hybridization, synaptogenesis

\footnotetext{
Abbreviations: AON, anterior olfactory nucleus; $\mathrm{BLA}$, basolateral amygdala; $\mathrm{CPu}$, caudate putamen; Npas4, neuronal PAS domain-containing protein 4; P, postnatal day; VGCC, voltage-gated calcium channels.
} 


\section{INTRODUCTION}

Npas4 is a member of the PAS family of proteins, a family of signal transduction molecules characterized by a conserved basic-helix-loop-helix motif and pas domain. Pas family proteins regulate a variety of biologically critical pathways, including those that are important for responses to external stimuli in adults and during development ( $\mathrm{Gu}$ et al., 2000). In the brain, Npas4 acts as an early-response transcription factor that, when induced by excitatory neuronal activity, regulates the formation of inhibitory synapses onto excitatory and inhibitory neurons (Lin et al., 2008; Coutellier et al., 2012; Bloodgood et al., 2013). By regulating inhibitory synapse numbers, Npas4 can affect the excitatory-inhibitory balance within neural circuits (Spiegel et al., 2014). Imbalances in excitatory and inhibitory activity have been implicated in several neurological disorders (Gao and Penzes, 2015), and thus, Npas4 may be a candidate gene for conditions such as anxiety, autism, bipolar depression and cognitive disorders, which often manifest during development (Jaehne et al., 2015).

In the adult mammalian brain, Npas4 mRNA is found in limbic structures, cortex, and striatum (Moser et al., 2004; Ooe et al., 2004; Shamloo et al., 2006). Although basal brain expression is low, it is rapidly upregulated in response to stimuli causing intense excitatory activity suggesting a role in cortical plasticity (Flood et al., 2004; Shamloo et al., 2006; Ploski et al., 2010; Bloodgood et al., 2013; Kaliszewska and Kossut, 2015). Npas4 is also upregulated in response to acute stress (Drouet et al., 2015), but in contrast, is down-regulated after chronic restraint stress or light deprivation (Yun et al., 2010; Karpova et al., 2010).

During development, activity-dependent synapse formation is critically important, especially during periods when sensory experience shapes cortical circuits (Hensch, 2005). In rodent cortical structures, inhibitory synapses begin to form during late prenatal and early postnatal development (Leinekugel, 2003), and are fine-tuned by neuronal activity (Chattopadhyaya et al., 2004). Despite the potentially important role of Npas4 in establishing inhibitory synapses, little is known about the spatial and temporal expression of Npas4 in the postnatal mammalian brain. In this study, using highly sensitive isotopic in situ hybridization, we determined the expression pattern of Npas 4 mRNA in postnatal and adult mouse brain under basal, non-challenged conditions.

\section{MATERIALS AND METHODS}

\section{Animals and Tissue Preparation}

All animals were handled and housed in accordance with the rules stipulated by the Texas A\&M University Animal Care Committee. C57BL/6 mice were housed in an animal care facility at $22-25^{\circ} \mathrm{C}$ with a 12 -h light/dark cycle and ad libitum food and water. Dams were allowed to give birth in the Texas A\&M University vivarium, and pups were nursed until postnatal day 21 (P21). Both male and female pups were used for experimentation but were not sexed for experiments. Only male mice were used at P60. At postnatal days (P)1, $3,5,8,13,21$, and 60 (young adult), pups and adults were brought to the lab in the morning, decapitated and their brains rapidly removed. Whole brains were immediately submerged in isopentane held at $-20^{\circ} \mathrm{C}$ for $30 \mathrm{~s}$, then stored at $-80^{\circ} \mathrm{C}$. $20 \mu \mathrm{m}$ coronal sections were taken through the brain using a Microtome cryostat (MICROM International $\mathrm{GmbH}$ ) kept at $-20^{\circ} \mathrm{C}$, and thaw-mounted on slides coated in poly-L-lysine (Sigma Chemical, St. Louis, MO, USA). Slides were fixed in $6 \%$ formaldehyde in $0.1 \mathrm{M}$ Phosphate Buffer solution (PB), then rinsed twice in $0.1 \mathrm{M} \mathrm{PB}$, once in $\mathrm{ddH}_{2} \mathrm{O}$, dried and stored at $-20^{\circ} \mathrm{C}$.

\section{In situ Hybridization}

An ${ }^{35}$ S-UTP-labeled cRNA probe for Npas4 was synthesized using a 762 bp. cDNA template, which was constructed via RT-PCR using forward (AGT GGC AGC ACT ACC TGG ATT TCT) and reverse (TCA GAG TTT AGC TGC TGG CGA AGA) primers (Integrated DNA Technologies), resulting in a final PCR product length of $762 \mathrm{bp}$. The PCR product was subcloned into a pPCR-Script AMP SK (+) plasmid (Stratagene, La Jolla, CA, USA). Sequencing results verified the correct sequence for mouse Npas4 mRNA. The plasmid was linearized via restriction enzyme digest, and cRNA probes were synthesized in the sense and antisense orientation with $\mathrm{T} 3$ and $\mathrm{T} 7$ RNA polymerase, respectively (Ambion, Austin, TX, USA) in the presence of ${ }^{35} \mathrm{~S}-\mathrm{UTP}$ (PerkinElmer, Boston, MA, USA). Non-specific hybridization was determined with the sense probe, and showed no or negligible hybridization signal (Figure, 1A insert). Specific hybridization was detected with the anti-sense probe and showed the expected expression pattern in adult mouse brain sections.

In situ hybridization was performed as previously described (Winzer-Serhan et al., 1999). Briefly, slides containing brain slices were pretreated for $10 \mathrm{~min}$ with protease $\mathrm{K}(0.1 \mu \mathrm{g} / \mathrm{mL})$, acetylated, dehydrated in graded ethanols and dried in a cold air stream. Slides were then incubated overnight at $60^{\circ} \mathrm{C}$ with the ${ }^{35} \mathrm{~S}$-UTP-labeled cRNA probe in hybridization solution (50\% formamide, $10 \%$ dextran sulfate, $500 \mu \mathrm{g} / \mathrm{ml}$ tRNA, $10 \mathrm{mM}$ dithiothreitol, $0.3 \mathrm{M} \mathrm{NaCl}, 10$ mMTris, $\mathrm{pH}$ 8.0, and $1 \mathrm{mM}$ EDTA, $\mathrm{pH} 8.0$ ). The next day, the slides were washed with RNAse A $(10 \mu \mathrm{g} / \mathrm{mL}$ Fisher Scientific, Pittsburgh, PA, USA) to remove any unbound RNA, and salinity was adjusted using decreasing concentrations of standard sodium citrate buffer (SSC). Sections were then dehydrated, dried, and apposed to Kodak BioMax-MR film (Fisher Scientific, Pittsburgh, PA, USA) along with $\left[{ }^{14} \mathrm{C}\right]-$ standards of known radioactivity (Amersham Bioscience, Buckinghamshire, England) where they were exposed at $4^{\circ} \mathrm{C}$.

For darkfield images, slides were dipped in Kodak autoradiography NTB emulsion (VWR, West Chester, PA, USA) and exposed at $4^{\circ} \mathrm{C}$. Slides were developed in Kodak D-19, fixed in Kodak Professional fixer (Fisher Scientific, Pittsburgh, 
PA, USA), counter-stained with Cresyl-Violet and cover slipped with DPX mounting medium (Fluka, Ronkonkoma, NY, USA).

\section{Analysis}

A computer-based image analysis system (MCID, Imaging Research Inc., St. Catherine, Canada; now InterFocus Imaging Ltd., UK) was used to analyze ${ }^{35}$ S-hybridization signals from autoradiograms. For quantification of the hybridization signal, ${ }^{14} \mathrm{C}$-standards were used to construct a calibration curve comparing relative optical density (ROD) to radioactivity in $\mathrm{nCi} / \mathrm{g}$. Hybridization intensities was calculated by converting ROD for antisense probes into $\mathrm{nCi} / \mathrm{g}$ as a measure of expression intensity, which was then adjusted for decay based on the ${ }^{35} \mathrm{~S}$ calibration date. Mean values of expression intensity and standard errors were calculated.

Microscopic analysis of the mRNA hybridization signals was performed using an Olympus BX1 microscope equipped for light- and dark-field microscopy. Images were taken with a DP7-1 digital camera (DP manager, Leeds Instruments, Irving, TX, USA).

\section{RESULTS}

\section{Adult Expression of Npas4 mRNA}

Npas4 mRNA expression in adult mouse brain was mostly restricted to telencephalic structures (Figure 1). The highest hybridization intensity was detected in the $\mathrm{AON}$, piriform cortex, frontal cortex, cingulate cortex, auditory cortex and hippocampus. Moderate expression was found in somatosensory and visual cortices. Low levels of expression were detected in the dorsal caudate putamen $(\mathrm{CPu})$, amygdala and in retrosplenial cortex. Most areas of the diencephalon or mesencephalon exhibited no expression, with the exception of the arcuate and paraventricular nucleus (PVN) of the hypothalamus.

\section{Npas4 mRNA Expression During Postnatal Development}

Npas4 expression was low in neonates (P1 and P3), and hybridization signals were only detected in the $\mathrm{CPu}$ and AON (data not shown). At P5, strong signal was located in the AON, where it remained high into adulthood, and moderate expression was found in the piriform cortex and $\mathrm{CPu}$ (Figure 2A). At the beginning of the second postnatal week (P8), low hybridization signal was also detected in a superficial layer of primary motor (M1) cortex, and in the pyramidal layer of dorsal and ventral hippocampus (Figure 2B). However, expression remained low in other cortical areas at P8. During the second and third postnatal week, signal intensities strongly increased in neocortex, cingulate cortex and in the pyramidal layer of CA1 and CA3 hippocampus, reaching adult levels of expression intensities (Figures 2C,D, 3). In the amygdala and septum, expression remained low. In general, areas that exhibited expression during postnatal development were the same that exhibited expression in adult brain areas. The exception was the $\mathrm{CPu}$, where transient hybridization signal was found in ventral regions during early postnatal development. However, with increasing postnatal age, the expression became restricted to the dorsal $\mathrm{CPu}$ with little or no expression in ventral $\mathrm{CPu}$ (Figures 2, 3A,B).

\section{Darkfield Analysis Of Npas4 mRNA Expression}

Darkfield microscopy revealed that most Npas4 mRNA was located in scattered cells in forebrain structures (Figures 4A-F). At P21, Npas4 mRNA expression was detected in scattered cells in the lateral septum and basolateral amygdala (BLA; Figures 4E,F). In contrast, expression in the PVN was more homogeneous (Figure 4G). In P13 neocortex, the number of cells exhibiting Npas4 expression was highest in layer VI and IV as shown for primary visual cortex (Figure 5A). At P21, Npas4expressing cells were found primarily in layers I-IV and VI, with only a few cells detected in layer V (Figure 5B). By P60, Npas4 mRNA expression was reduced to some scattered cells in layers II-VI (Figure 5C). However, in auditory cortex, expression remained more pronounced in adults.

In the hippocampus, NPas4 mRNA was detected in CA1, CA2, CA3 and DG with similar expression levels in dorsal and ventral hippocampus (Figure 6). Moderate NPas4 mRNA hybridization signal was evenly distributed in pyramidal cells of the stratum (s.) pyramidale in subfield CA1 and CA2 (Figure 6B), whereas in CA3 s. pyramidale, numerous individual cells with robust signal were found (Figure 6D). In the DG, NPas4 mRNA was found in a few cells located in the granule cell layer and in the hilus region (Figure 6C). Almost no expression was detected in cells located in s. oriens or radiatum of either CA1 or CA3, or in the molecular layer of the DG.

\section{DISCUSSION}

This study describes the expression pattern of the transcriptional regulator Npas4 in postnatal and adult male mouse brains using highly sensitive isotopic in situ hybridization with an Npas4 specific cRNA probe. In adults and during development Npas4 expression was almost exclusively found in areas of the telencephalon, including neocortex, hippocampus, basal ganglia and olfactory bulb, with some expression also detected in the septum and amygdala. In contrast, Npas4 expression in non-telencephalic brain areas including the diencephalon and mesencephalon was very low and restricted to the hypothalamic arcuate and PVN. In most of telencephalic areas, Npas4 mRNA expression started during the first and second postnatal week and increased in intensity with little change in spatial distribution compared to adults. The exception was the $\mathrm{CPu}$, where Npas4 expression became increasingly restricted to the dorsal $\mathrm{CPu}$.

\section{Expression of Npas4 in the Adult Brain}

Previous studies using northern blot analysis had detected Npas4 mRNA (then called LE-PAS or Nxf) in frontal and entorhinal cortices, hippocampus and olfactory bulb (Moser et al., 2004). Studies using non-radioactive in situ hybridization found additional mRNA expression in hippocampus and neocortex 

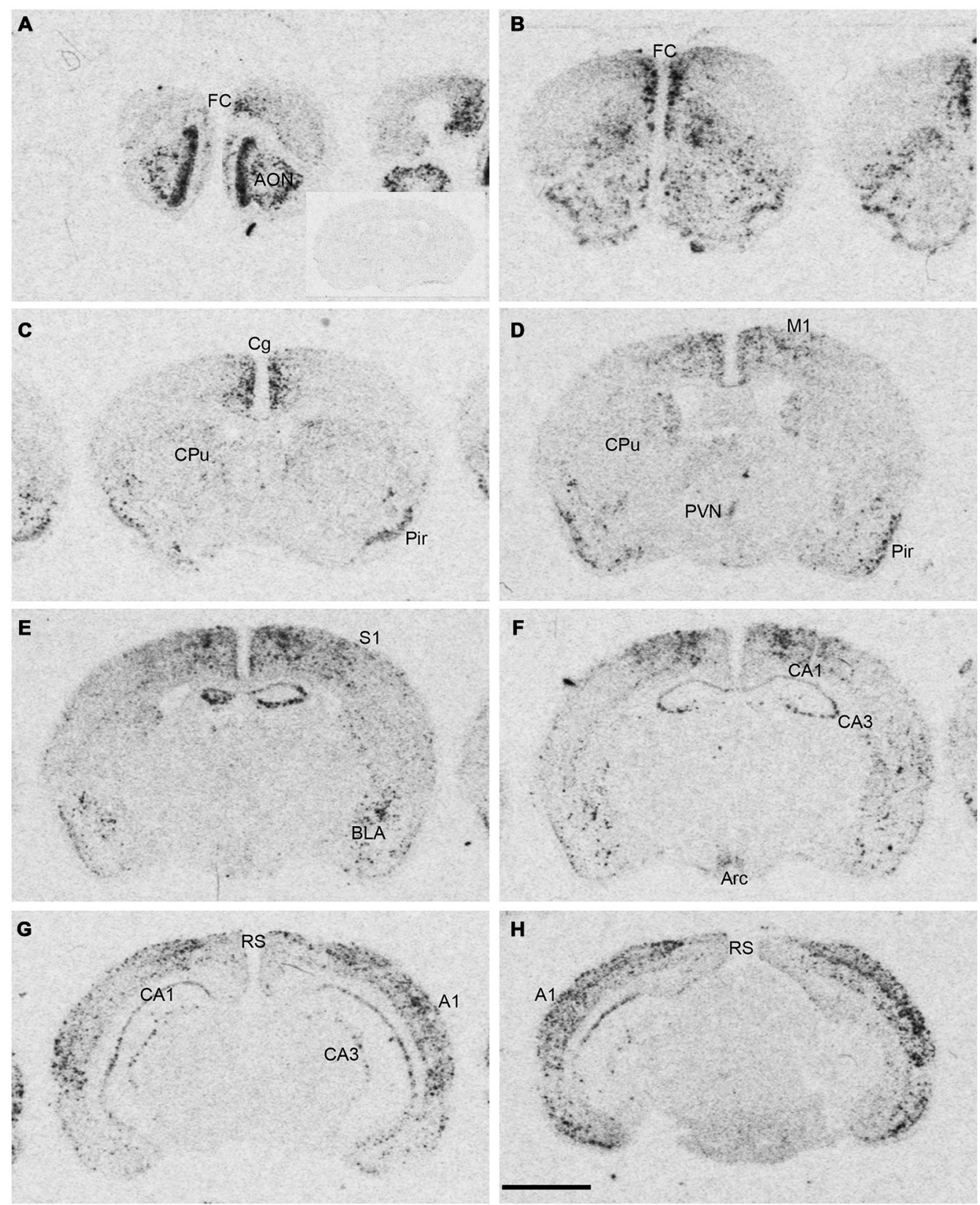

FIGURE 1 | Npas4 mRNA expression in adult mouse brains. Autoradiographic images of Npas4 mRNA hybridization in coronal sections of (A,B) the anterior olfactory nucleus (AON) and frontal cortex (FC), (C,D) caudate putamen (CPu), (E,F) dorsal hippocampus, $\mathbf{( G , H ) ~ v e n t r a l ~ h i p p o c a m p u s . ~ I n s e r t ~ i n ~ ( A ) ~ r e p r e s e n t s ~ a n ~}$ adult mouse brain section at the level of the dorsal hippocampus hybridized with the Npas4 sense probe. Abbreviations: A1, primary auditory cortex; AON, anterior olfactory nucleus; Arc, arcuate nucleus; BLA, basolateral amygdala nucleus; Cg, cingulate cortex; DG, dentate gyrus; FC, frontal cortex; M1, primary motor cortex; Pir, piriform cortex; PVN, paraventricular hypothalamic nucleus; RS, retrosplenial cortex; S1 primary somatosensory cortex; V1, primary visual cortex. Scale $\mathrm{bar}=2 \mathrm{~mm}$.

(Lin et al., 2008; Moser et al., 2004). In addition to expression in hippocampus and neocortex, Npas4 mRNA transcripts were also detected in thalamus and striatum of adult rat brain (Ooe et al., 2004). To verify the distribution pattern of Npas4 mRNA in adult mouse brain, we used isotopic in situ hybridization with an antisense cRNA probe specific for Npas4, allowing for high spatial resolution and detection of mRNA transcripts in single cells (Winzer-Serhan et al., 1999). With this approach, we verified Npas4 expression in telencephalic areas including the olfactory system, neocortex, hippocampus and the amygdala, and confirmed striatal expression in mouse brain. In contrast to results from rat (Ooe et al., 2004), expression in the diencephalon 


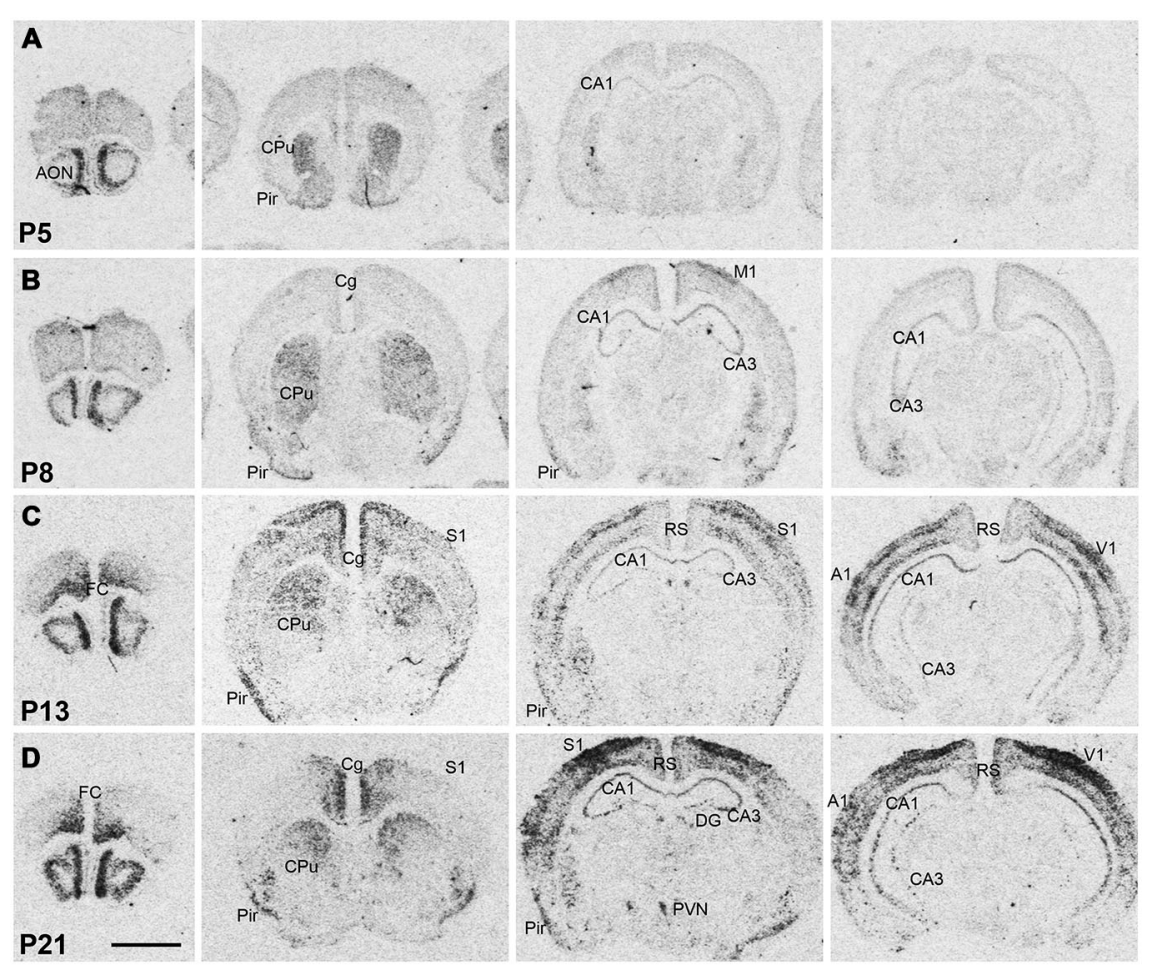

FIGURE 2 | Npas4 mRNA expression in postnatal mouse brains. Autoradiographic images of Npas4 mRNA hybridization in coronal sections of the anterior olfactory nucleus (AON) and frontal cortex, caudate putamen (CPu), dorsal hippocampus, and ventral hippocampus at (A) postnatal day (P)5, (B) P8, (C) P13, (D) P21. Abbreviations: see Figure 1. Scale bar $=2 \mathrm{~mm}$.

was limited to the hypothalamic PVN and arcuate nucleus. This difference might be explained by differential expression of NPas 4 mRNA between rodent species. However, in the unchallenged mouse brain used in this study, Npas 4 mRNA was mostly expressed in regions of the telencephalon, and exhibits no or very limited expression in the diencephalon and mesencephalon.

NPas4 mRNA was not uniformly expressed in all neurons of the telencephalon, but was restricted to scattered cells which exhibited strong hybridization signal. The exceptions were the olfactory bulb and CA1 s. pyramidale, areas that exhibited strong or moderate expression in most neurons, which was not detected with the sense probe. The scattered distribution of cells with intense signal suggests that Npas4 expression may be localized in GABAergic neurons, in temporarily active excitatory neurons, or in both, but the identity of these cells still needs to be determined. Certain areas harbored more Npas4 positive neurons than others. For example, increased numbers were detected in frontal, cingulate and auditory cortices, which may suggest excitatory activity strong enough to maintain strong Npas4 expression. Particularly striking was the strong Npas4 hybridization signal in a subset of neurons located in the hippocampal CA3 s. pyramidale and in the granule layer of the DG. There is a sub-population of CA3 pyramidal cells which originate from early born glutamatergic neurons. These neurons are highly connected and are considered central nodes in the functional organization of hippocampal networks (Marissal et al., 2012).
Thus, it is possible that these neurons are constitutively active and therefore, continuously express Npas 4 mRNA. However, further studies need to verify the identity of these cells expressing high levels of Npas4 and to correlate neuronal activity to Npas4 expression levels under basal conditions in the mature male brain.

A previous report found expression of Npas4 in interneurons located in s. oriens and radiatum (Moser et al., 2004). However, in this study, there was very little or no expression in cells located in s. oriens and radiatum The reasons for this discrepancy is not clear, but since Npas4 is an immediate early gene that is easily induced within minutes by a variety of stimuli (Flood et al., 2004; Shamloo et al., 2006; Ramamoorthi et al., 2011), it is possible that differential handling and/or housing conditions prior to sacrificing the animals could account for this difference.

In addition to the expression in the hippocampus and neocortex, Npas4 expression was robust in the olfactory system, including olfactory bulb, AON and piriform cortex starting in neonates and persisting into adulthood. These areas have high GABAergic tone and high densities of GABAergic synapses which are strongly involved in the regulation of excitatory activity (Ribak et al., 1977). Npas4 might have an important role in the regulation of inhibitory synaptic plasticity, and in the maintenance of the excitatory/inhibitory balance in the olfactory system, an idea that is supported by recent findings that Npas4 expression is rapidly increased by odor presentation 

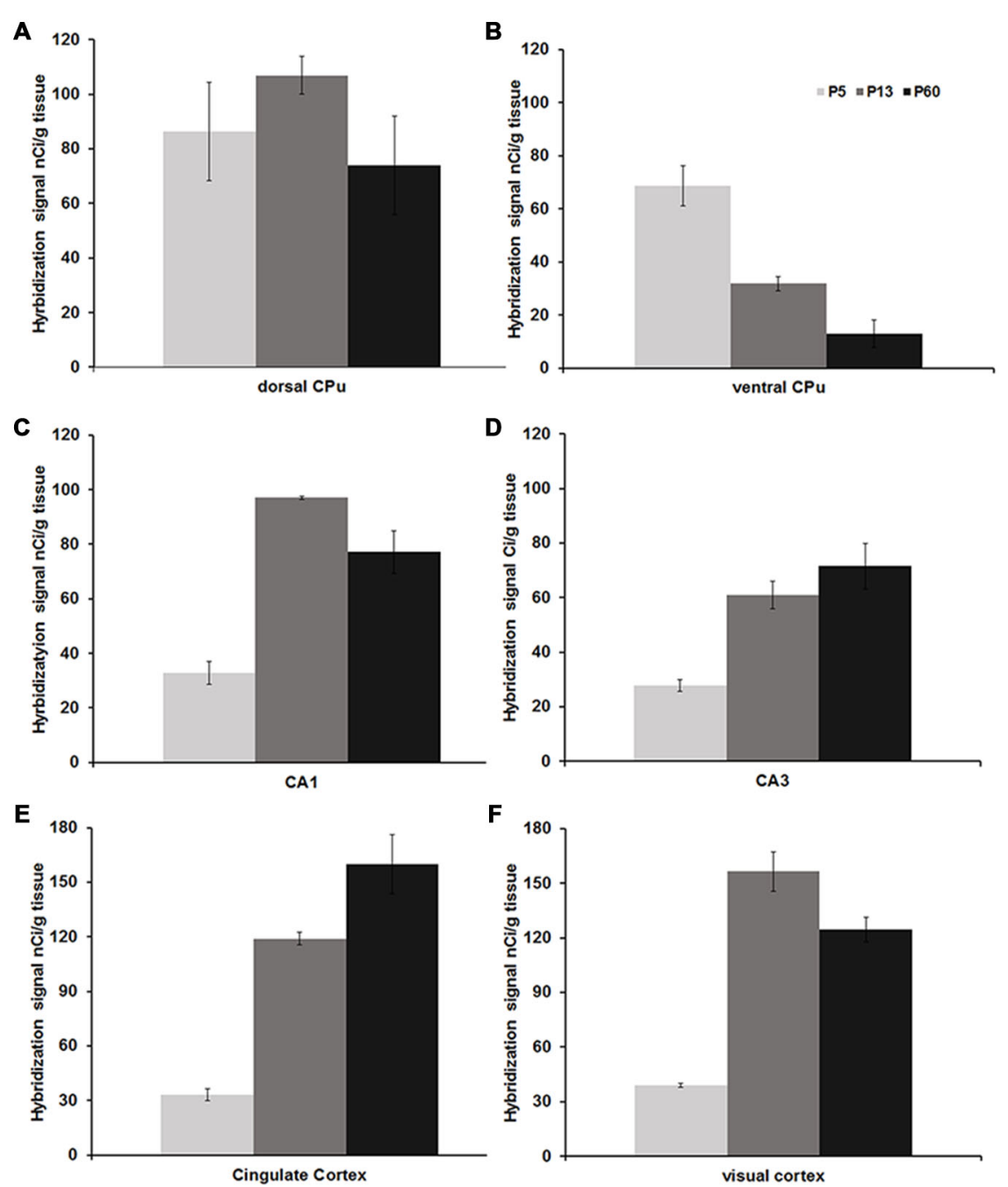

FIGURE 3 | Mean Npas4 hybridization intensities. Expression in (A) dorsal caudate, (B) ventral caudate, (C) CA1 stratum pyramidale, (D) CA3 stratum pyramidale, $(\mathbf{E})$ cingulate cortex, $\mathbf{( F )}$ primary visual cortex. Light gray bars = postnatal day $(\mathrm{P}) 5$, dark gray bars $=\mathrm{P} 13$, black bars $=\mathrm{P} 60 ; n=3$. Error bars $=$ standard error.

(Bepari et al., 2012). In addition, a functional role of Npas4 in the developing sensory olfactory system has recently been described (Yoshihara et al., 2014). Interestingly, in that study, the majority of Npas4 positive neurons represent GAD67 positive interneurons indicating a role in inhibitory synapse formation in inhibitory neurons.

\section{Developmental Expression of Npas4}

Npas4 mRNA expression fully emerged during postnatal development. Prior to birth, Npas4 mRNA hybridization signal was very low, when evaluated in embryonic brain sections from gestational age 18 and 21 embryos (data not shown). There was a general pattern of increasing expression intensity during the first two postnatal weeks in telencephalic areas, which established the adult-like pattern. The olfactory system exhibited robust expression, shortly after birth, with other brain areas following thereafter. A similar expression pattern has been described in other vertebrate species including the developing zebrafish brain (Klari ć et al., 2014) GABAergic and glutamatergic.

During late prenatal and early postnatal development, the first functional synapses formed in cortical structures are GABAergic, followed by glutamatergic synapses, which are initially silent during the neonatal period (Ben-Ari et al., 1997; Tyzio et al., 1999; Hennou et al., 2002; Gozlan and Ben-Ari, 2003; Voigt et al., 2005; Ben-Ari, 2006). Thus, early developmental GABAergic synaptogenesis takes places in the absence of excitatory glutamatergic synaptic activity, and we expected to see onset of Npas4 expression during the developmental period when GABAergic synapses are initially formed. However, in most cortical areas that was not the case. Instead, cortical Npas4 mRNA expression was very low in neonates, suggesting that the initial formation of GABAergic synapses is independent of Npas4 activity. This is in line with studies showing that Npas4 knockout mice develop normal inhibitory synapses (Lin et al., 


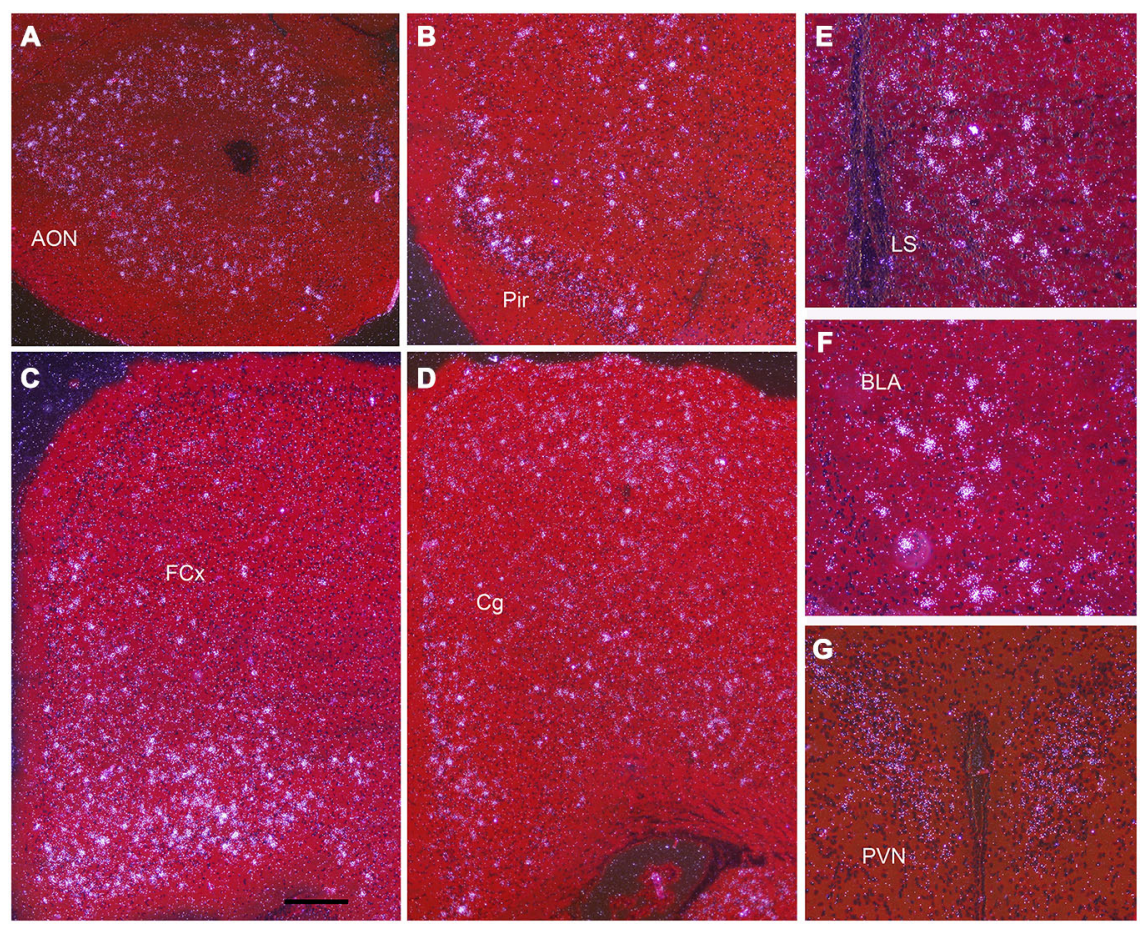

FIGURE 4 | Npas4 mRNA expression at postnatal day (P)21. Darkfield photomicrographs of hybridization in (A) the anterior olfactory nucleus (AON), (B) pirifrom corex (Pir), (C) Frontal cortex (FCX), (D) cingulate cortex (Cg), (E) lateral septum (LS), (F) basolateral amygdala (BLA), (G) paraventricular nucleus (PVN). Scale bar = $250 \mu \mathrm{m}$ in (A-D), $100 \mu \mathrm{m}$ in (E-G).

2008). However, it remains to be seen if an excitatory stimulus could drive Npas4 expression in the immature brain, and thereby affect GABAergic synapse formation during the perinatal period.

In most neocortical areas, Npas 4 mRNA expression strongly increased by the end of the second postnatal week, especially in layers IV and VI of sensory cortices (see Figure 2). It has been shown that induction of Npas4 mRNA depends on triad of NMDA receptor, AMPA receptor and voltage-gated calcium channel (VGCC) activation (Lin et al., 2008). This would suggest that mature glutamatergic synaptic transmission consisting of NMDA + AMPA receptor mediated components needs to be in place in order to effectively induce Npas4 mRNA expression. With increasing postnatal age, the relative NMDA/AMPA receptor ratio decreases, so that by the end
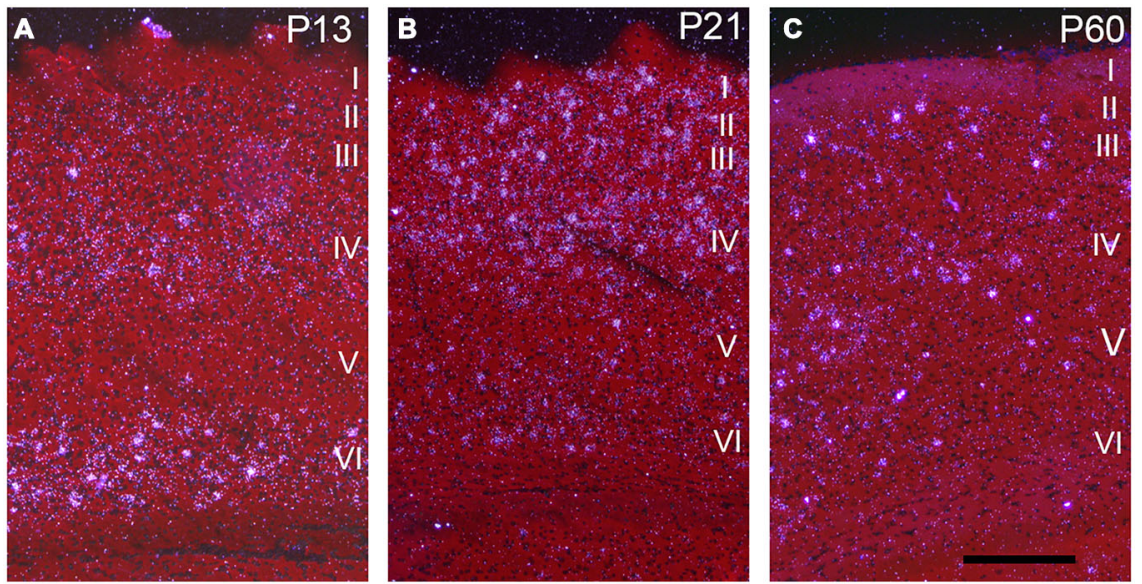

FIGURE 5 | Npas4 mRNA expression in primary visual cortex. Darkfield photomicrographs of hybridization in coronal sections at (A) postnatal day P13, (B) P21 and (C) P60. The Roman numerals indicate the cortical layers in primary visual cortex. Scale bar $=250 \mu \mathrm{m}$. 

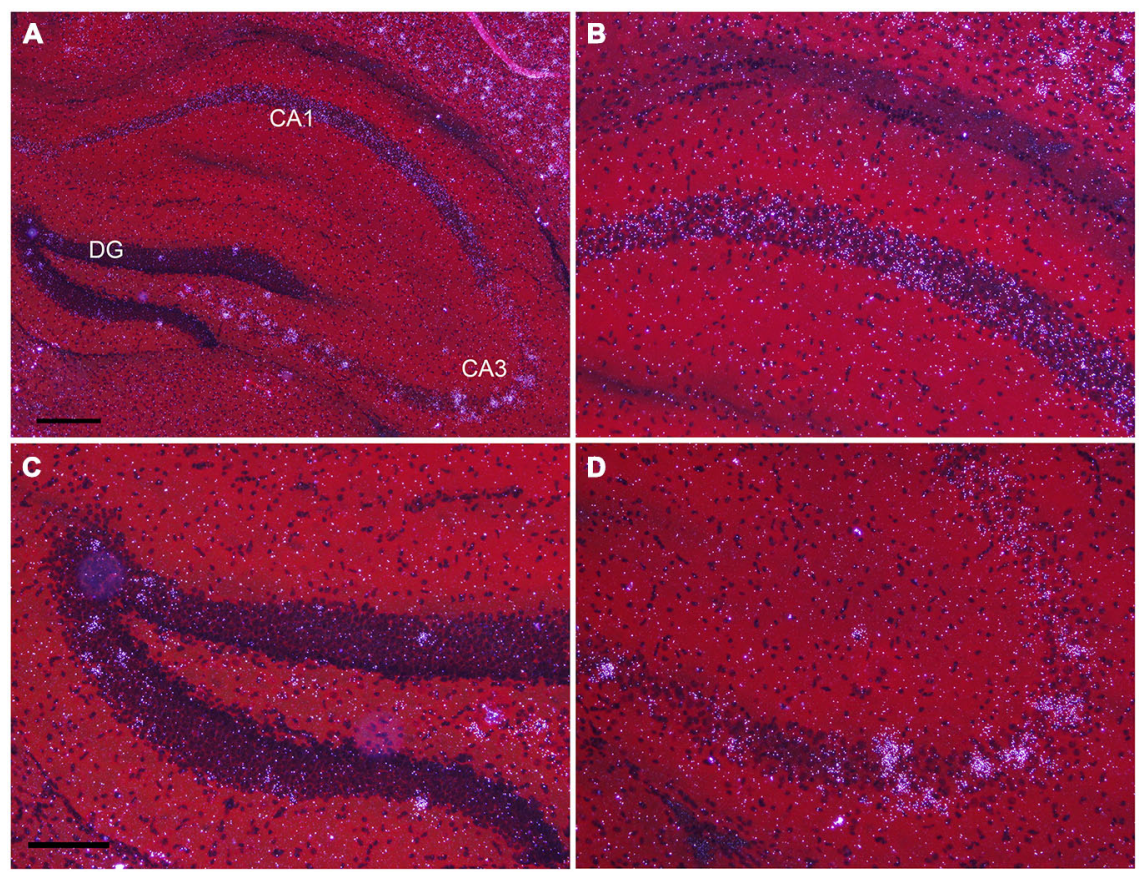

FIGURE 6 | Npas4 mRNA expression in dorsal hippocampus (A) Darkfield photomicrographs of hybridization in the dorsal hippocampus, (B) in the CA1, (C) in dentate gyrus, (D) in CA3 at postnatal day 21. Scale bars: in (A) $=250 \mu \mathrm{m}$, in (C) $=125 \mu \mathrm{m}$ (applies to B-D).

of the second postnatal week mature NMDA + AMPA receptor-mediated excitatory synaptic transmission is established (Golshani and Jones, 1999; Brill and Huguenard, 2008). This coincides with increased activity of thalamocortical axonal projections relaying sensory information to the cortex by the end of the second postnatal week (Agmon and O'Dowd, 1992, Feldmeyer and Radnikow, 2009; Dorrn et al., 2010). This postnatal time point also represents a period of profound transformational changes in cortical synaptic network activities (Micheva and Beaulieu, 1997; Lendvai et al., 2000; Stern et al., 2001; Maravall et al., 2004), culminating in the functional maturation of large-scale cortical network activity around P13 in rodents (Quairiaux et al., 2011), which correlates with the sharp increase in Npas4 mRNA expression at P13 in sensory cortices detected in this study. Thus, although correlative, Npas 4 mRNA expression seems to follow the maturation of excitatory cortical circuits, perhaps marking the beginning of activity-driven inhibitory synapse formation, which could explain the large increase in the density of inhibitory synapses at the beginning of the third postnatal week (De Felipe et al., 1997).

In the hippocampus, developmental Npas4 expression exhibits a similar pattern as seen in the cortex by temporally following the maturation of excitatory synapses. Comparable to neocortical development, GABAergic synapses appear first, followed by glutamatergic ones, and most excitatory pyramidal neurons are silent, and have no or a very small AMPA receptor component in the neonatal hippocampus, (Durand et al., 1996; Hsia et al., 1998; Tyzio et al., 1999; Hennou et al., 2002). The maturation of excitatory glutamatergic synaptic responses takes place in the hippocampus during the first postnatal week (Hsia et al., 1998; Leinekugel, 2003). Npas4 mRNA expression greatly increased between $\mathrm{P} 5$ and P8 in CA1 and CA3 s. pyramidale, supporting the notion that mature AMPA + NMDA receptor mediated excitatory transmission drives Npas4 mRNA expression. It remains to be determined if aberrant excitatory activity, perhaps caused by neonatal seizures, could induce Npas 4 mRNA expression in the hippocampus at an earlier age and alter the formation of inhibitory synapses.

\section{CONCLUSION}

Npas4 is a transcriptional regulator that could have a significant regulatory role in setting up cortical circuits and shaping the excitatory/inhibitory balance in the developing brain. Adult expression of Npas4 is restricted to telencephalic regions where scattered cells with high levels of expression are found. In the developing mouse brain, Npas4 mRNA distribution exhibits a similar spatial distribution pattern. The onset of Npas4 mRNA expression during the second and third postnatal week seems to correlate with the maturation of excitatory synapses from functionally silent into active ones as AMPA receptors become increasingly incorporated in postsynaptic membranes. It remains to be determined if an increase in neuronal excitatory activity is responsible for the increase in Npas4 expression during postnatal development. However, this information could serve as a starting point to explore the potential roles of Npas4 during development, which in turn could lend insight into some of the mechanisms of activity-dependent brain development. 


\section{AUTHOR CONTRIBUTIONS}

JCD: conducted the experiments, generated a riboprobe specific for Npas4, analyzed the results, prepared the manuscript. GSS: conducted the experiments, helped in writing the manuscript. UHWS: Planned and oversaw the project, analyzed the results, prepared the manuscript.

\section{REFERENCES}

Agmon, A., and O’Dowd, D. K. (1992). NMDA receptor-mediated currents are prominent in the thalamocortical synaptic response before maturation of inhibition. J. Neurophysiol. 68, 345-349.

Ben-Ari, Y. (2006). Basic developmental rules and their implications for epilepsy in the immature brain. Epileptic Disord. 8, 91-102.

Ben-Ari, Y., Khazipov, R., Leinekugel, X., Caillard, O., and Gaiarsa, J. L. (1997). GABAA, NMDA and AMPA receptors: a developmentally regulated 'ménage à trois'. Trends Neurosci. 20, 523-529. doi: 10.1016/s0166-2236(97) 01147-8

Bepari, A. K., Watanabe, K., Yamaguchi, M., Tamamaki, N., and Takebayashi, H. (2012). Visualization of odor-induced neuronal activity by immediate early gene expression. BMC Neurosci. 13:140. doi: 10.1186/1471-2202-13-140

Bloodgood, B. L., Sharma, N., Browne, H. A., Trepman, A. Z., and Greenberg, M. E. (2013). The activity-dependent transcription factor NPAS4 regulates domainspecific inhibition. Nature 503, 121-125. doi: 10.1038/nature12743

Brill, J., and Huguenard, J. R. (2008). Sequential changes in AMPA receptor targeting in the developing neocortical excitatory circuit. J. Neurosci. 28, 13918-13928. doi: 10.1523/JNEUROSCI.3229-08.2008

Chattopadhyaya, B., Di Cristo, G., Higashiyama, H., Knott, G. W., Kuhlman, S. J., Welker, E., et al. (2004). Experience and activity-dependent maturation of perisomatic GABAergic innervation in primary visual cortex during a postnatal critical period. J. Neurosci. 24, 9598-9611. doi: 10.1523/jneurosci.1851-04.2004

Coutellier, L., Beraki, S., Ardestani, P. M., Saw, N. L., and Shamloo, M. (2012). Npas4: a neuronal transcription factor with a key role in social and cognitive functions relevant to developmental disorders. PLoS One 7:e46604. doi: 10. 1371/journal.pone.0046604

De Felipe, J., Marco, P., Fairén, A., and Jones, E. G. (1997). Inhibitory synaptogenesis in mouse somatosensory cortex. Cereb. Cortex 7, 619-634. doi: 10.1093/cercor/7.7.619

Dorrn, A. L., Yuan, K., Barker, A. J., Schreiner, C. E., and Froemke, R. C. (2010). Developmental sensory experience balances cortical excitation and inhibition. Nature 465, 932-936. doi: 10.1038/nature09119

Drouet, J. B., Fauvelle, F., Maunoir-Regimbal, S., Fidier, N., Maury, R., Peinnequin, A., et al. (2015). Differences in prefrontal cortex GABA/glutamate ratio after acute restraint stress in rats are associated with specific behavioral and neurobiological patterns. Neuroscience 285, 155-165. doi: 10.1016/j. neuroscience.2014.10.058

Durand, G. M., Kovalchuk, Y., and Konnerth, A. (1996). Long-term potentiation and functional synapse induction in developing hippocampus. Nature 381, 71-75. doi: 10.1038/381071a0

Feldmeyer, D., and Radnikow, G. (2009). Developmental alterations in the functional properties of excitatory neocortical synapses. J. Physiol. 587, 1889-1896. doi: 10.1113/jphysiol.2009.169458

Flood, W. D., Moyer, R. W., Tsykin, A., Sutherland, G. R., and Koblar, S. A. (2004). Nxf and Fbxo33: novel seizure-responsive genes in mice. Eur. J. Neurosci. 20, 1819-1826. doi: 10.1111/j.1460-9568.2004.03646.x

Gao, R., and Penzes, P. (2015). Common mechanisms of excitatory and inhibitory imbalance in schizophrenia and autism spectrum disorders. Curr. Mol. Med. 15, 146-167. doi: 10.2174/1566524015666150303003028

Golshani, P., and Jones, E. G. (1999). Synchronized paroxysmal activity in the developing thalamocortical network mediated by corticothalamic projections and "silent" synapses. J. Neurosci. 19, 2865-2875. doi: 10.1002/(sici)10974695(199910)41:1<92::aid-neu12>3.3.co;2-1

Gozlan, H., and Ben-Ari, Y. (2003). Interneurons are the source and the targets of the first synapses formed in the rat developing hippocampal circuit. Cereb. Cortex 13, 684-692. doi: 10.1093/cercor/13.6.684

\section{ACKNOWLEDGMENTS}

JCD received funding from Texas Consortium in Behavioral Neuroscience Predoctoral Fellowship, and a Texas Brain and Spine Institute Graduate Fellowship; UHWS received funding a Bridge Grant from the Texas A\&M Health Science Center.

Gu, Y. Z., Hogenesch, J. B., and Bradfield, C. A. (2000). The PAS superfamily: sensors of environmental and developmental signals. Annu. Rev. Pharmacol. Toxicol. 40, 519-561. doi: 10.1146/annurev.pharmtox. 40.1.519

Hennou, S., Khalilov, I., Diabira, D., Ben-Ari, Y., and Gozlan, H. (2002). Early sequential formation of functional $\mathrm{GABA}(\mathrm{A})$ and glutamatergic synapses on CA1 interneurons of the rat foetal hippocampus. Eur. J. Neurosci. 16, 197-208. doi: 10.1046/j.1460-9568.2002.02073.x

Hensch, T. K. (2005). Critical period plasticity in local cortical circuits. Nat. Rev. Neurosci. 6, 877-888. doi: 10.1038/nrn1787

Hsia, A. Y., Malenka, R. C., and Nicoll, R. A. (1998). Development of excitatory circuitry in the hippocampus. J. Neurophysiol. 79, 2013-2024.

Jaehne, E. J., Klarić, T. S., Koblar, S. A., Baune, B. T., and Lewis, M. D. (2015). Effects of Npas4 deficiency on anxiety, depression-like, cognition and sociability behavior. Behav. Brain Res. 281, 276-282. doi: 10.1016/j.bbr.2014. 12.044

Kaliszewska, A., and Kossut, M. (2015). Npas4 expression in two experimental models of the barrel cortex plasticity. Neural Plast. 2015:175701. doi: 10. $1155 / 2015 / 175701$

Karpova, N. N., Rantamaki, T., Di Lieto, A., Lindemann, L., Hoener, M. C., and Castren, E. (2010). Darkness reduces BDNF expression in the visual cortex and induces repressive chromatin remodeling at the BDNF gene in both hippocampus and visual cortex. Cell. Mol. Neurobiol. 30, 1117-1123. doi: 10. 1007/s10571-010-9544-6

Klarić, T., Lardelli, M., Key, B., Koblar, S., and Lewis, M. (2014). Activitydependent expression of neuronal PAS domain-containing protein 4 (npas4a) in the developing zebrafish brain. Front. Neuroanat. 8:148. doi: 10.3389/fnana. 2014.00148

Leinekugel, X. (2003). Developmental patterns and plasticities: the hippocampal model. J. Physiol. Paris 97, 27-37. doi: 10.1016/j.jphysparis.2003.10.004

Lendvai, B., Stern, E. A., Chen, B., and Svoboda, K. (2000). Experience-dependent plasticity of dendritic spines in the developing rat barrel cortex in vivo. Nature 404, 876-881. doi: 10.1038/35009107

Lin, Y., Bloodgood, B. L., Hauser, J. L., Lapan, A. D., Koon, A. C., Kim, T. K., et al. (2008). Activity-dependent regulation of inhibitory synapse development by Npas4. Nature 455, 1198-1204. doi: 10.1038/nature07319

Maravall, M., Stern, E. A., and Svoboda, K. (2004). Development of intrinsic properties and excitability of layer $2 / 3$ pyramidal neurons during a critical period for sensory maps in rat barrel cortex. J. Neurophysiol. 92, 144-156. doi: 10.1152/jn.00598.2003

Marissal, T., Bonifazi, P., Picardo, M. A., Nardou, R., Petit, L. F., Baude, A., et al. (2012). Pioneer glutamatergic cells develop into a morpho-functionally distinct population in the juvenile CA3 hippocampus. Nat. Commun. 3:1316. doi: 10 $1038 /$ ncomms 2318

Micheva, K. D., and Beaulieu, C. (1997). Development and plasticity of the inhibitory neocortical circuitry with an emphasis on the rodent barrel field cortex: a review. Can. J. Physiol. Pharmacol. 75, 470-478. doi: 10.1139/y97-032

Moser, M., Knoth, R., Bode, C., and Patterson, C. (2004). LE-PAS, a novel Arntdependent HLH-PAS protein, is expressed in limbic tissues and transactivates the CNS midline enhancer element. Brain Res. Mol. Brain Res. 128, 141-149. doi: 10.1016/j.molbrainres.2004.06.023

Ooe, N., Saito, K., Mikami, N., Nakatuka, I., and Kaneko, H. (2004) Identification of a novel basic helix-loop-helix-PAS factor, NXF, reveals a Sim 2 competitive, positive regulatory role in dendritic-cytoskeleton modulator drebrin gene expression. Mol. Cell. Biol. 24, 608-616. doi: 10.1128/mcb.24.2. 608-616.2004

Ploski, J. E., Park, K. W., Ping, J., Monsey, M. S., and Schafe, G. E. (2010) Identification of plasticity-associated genes regulated by pavlovian fear 
conditioning in the lateral amygdala. J. Neurochem. 112, 636-650. doi: 10 . 1111/j.1471-4159.2009.06491.x

Quairiaux, C., Mégevand, P., Kiss, J. Z., and Michel, C. M. (2011). Functional development of large-scale sensorimotor cortical networks in the brain. J. Neurosci. 31, 9574-9584. doi: 10.1523/JNEUROSCI.599510.2011

Ramamoorthi, K., Fropf, R., Belfort, G. M., Fitzmaurice, H. L., McKinney, R. M., Neve, R. L., et al. (2011). Npas4 regulates a transcriptional program in CA3 required for contextual memory formation. Science 334, 1669-1675. doi: 10 . 1126/science. 1208049

Ribak, C. E., Vaughn, J. E., Saito, K., Barber, R., and Roberts, E. (1977). Glutamate decarboxylase localization in neurons of the olfactory bulb. Brain Res. 126, 1-18. doi: 10.1016/0006-8993(77) 90211-6

Shamloo, M., Soriano, L., von Schack, D., Rickhag, M., Chin, D. J., GonzalezZulueta, M., et al. (2006). Npas4, a novel helix-loop-helix PAS domain protein, is regulated in response to cerebral ischemia. Eur. J. Neurosci. 24, 2705-2720. doi: 10.1111/j.1460-9568.2006.05172.x

Spiegel, I., Mardinly, A. R., Gabel, H. W., Bazinet, J. E., Couch, C. H., Tzeng, C. P., et al. (2014). Npas4 regulates excitatory-inhibitory balance within neural circuits through cell-type-specific gene programs. Cell 157, 1216-1229. doi: 10. 1016/j.cell.2014.03.058

Stern, E. A., Maravall, M., and Svoboda, K. (2001). Rapid development and plasticity of layer 2/3 maps in rat barrel cortex in vivo. Neuron 31, 305-315. doi: 10.1016/s0896-6273(01)00360-9

Tyzio, R., Represa, A., Jorquera, I., Ben-Ari, Y., Gozlan, H., and Aniksztejn, L. (1999). The establishment of GABAergic and glutamatergic synapses on CA1 pyramidal neurons is sequential and correlates with the development of the apical dendrite. J. Neurosci. 19, 10372-10382.
Voigt, T., Opitz, T., and de Lima, A. D. (2005). Activation of early silent synapses by spontaneous synchronous network activity limits the range of neocortical connections. J. Neurosci. 25, 4605-4615. doi: 10.1523/jneurosci.3803-04. 2005

Winzer-Serhan, U. H., Broide, R. S., Chen, Y., and Leslie, F. M. (1999). Highly sensitive radioactive in situ hybridization using full length hydrolyzed riboprobes to detect alpha 2 adrenoceptor subtype mRNAs in adult and developing rat brain. Brain Res. Brain Res. Protoc. 3, 229-241. doi: 10. 1016/s1385-299x(98)00043-9

Yoshihara, S., Takahashi, H., Nishimura, N., Kinoshita, M., Asahina, R., Kitsuki, M., et al. (2014). Npas4 regulates Mdm2 and thus Dcx in experience-dependent dendritic spine development of newborn olfactory bulb interneurons. Cell Rep. 8, 843-857. doi: 10.1016/j.celrep.2014.06.056

Yun, J., Koike, H., Ibi, D., Toth, E., Mizoguchi, H., Nitta, A., et al. (2010). Chronic restraint stress impairs neurogenesis and hippocampus-dependent fear memory in mice: possible involvement of a brain-specific transcription factor Npas4. J. Neurochem. 114, 1840-1851. doi: 10.1111/j.1471-4159.2010. 06893.x

Conflict of Interest Statement: The authors declare that the research was conducted in the absence of any commercial or financial relationships that could be construed as a potential conflict of interest.

Copyright (C) 2015 Damborsky, Slaton and Winzer-Serhan. This is an open-access article distributed under the terms of the Creative Commons Attribution License (CC $B Y)$. The use, distribution and reproduction in other forums is permitted, provided the original author(s) or licensor are credited and that the original publication in this journal is cited, in accordance with accepted academic practice. No use, distribution or reproduction is permitted which does not comply with these terms. 